\title{
Doped Gelatin Films as a Model Matrix for Molecular Secondary Ion Mass Spectrometry Studies of Biological Soft Tissue
}

\author{
Greg Gillen \\ Gaithersburg, Maryland, USA \\ Steven M. Hues \\ Chemistry Division, Naval Research Laboratory, Washington, DC, USA
}

Surface and Microanalysis Science Division, National Institute of Standards and Technology,

\begin{abstract}
Porcine gelatin films doped with a number of biological compounds at various concentrations and prepared by spin-casting have been used as model biological tissue matrices for studying organic ion emission in molecular secondary ion mass spectrometry. For many compounds, portions of the working curves were found to be linear over several orders of magnitude in concentration. Detection limits for the analyzed compounds were in the parts per million range for several organic salt compounds but high $(0.1 w t \%)$ for others. Owing to the presence of a significant chemical background, the poorest detection limits were generally obtained from compounds with low molecular weights. Secondary ion yield matrix effects, indicated by a reduction in ionization efficiency at higher concentrations, were observed for several organic salt compounds. () Am Soc Mass Spectrom 1993, 4, 419-423)
\end{abstract}

A nalysis and imaging of physiologically important organic compounds in biological materials, such as tissue, by secondary ion mass spectrometry (SIMS) have become an area of much interest in recent years [1-3]. Progress in this area has been hampered by our poor understanding of molecular emission from the tissue matrix. A number of issues remain to be clarified: How severe is chemical background? What detection limits would be expected for different types of compounds? Are ion yields in molecular SIMS proportional to concentration? To examine these questions, it would be advantageous to have a tissue or tissuelike matrix that eliminates the problems associated with analysis of real tissue samples (i.e., gross heterogeneity and charging). One approach, often used for elemental analysis of biological tissue, is to use doped gelatin or Epon plastic as a tissue model [4-7]. Gelatin has several advantages: It is readily available and easy to prepare; its elemental composition is similar to that of tissue; it is compatible with the high vacuum of the SIMS instrument; and it can be doped with a variety of molecular species. Gelatin films for elemental analysis have typically been prepared by dropping the gelatin onto a metallic substrate and allowing it to air dry; however, this produces a

Address reprint requests to Greg Gillen, Surface and Microanalysis Science Division, National Institute of Standards and Technology, Gaithersburg, MD 20899. film that is nonuniform in thickness and very susceptible to charging during analysis. To minimize charging, the samples are typically overcoated with a conductive metallic layer. Although this causes no difficulties for elemental analysis, the analyte molecules in a molecular film would be completely fragmented by the penetrating ion beam during sputter removal of the metallic overlayer. In this study a new method is developed for preparing gelatin films using spin-casting, which produces films with superior homogeneity and minimal charging problems. These films allow us to study the emission of molecular species from a tissuelike matrix under well-controlled conditions. The linearity of the calibration curves and the detection limits for several different compound/gelatin mixtures are evaluated. Also, the possibility of using these gelatin films as standards for molecular imaging SIMS is demonstrated.

\section{Experimental}

Porcine gelatin A ( $2 \%$ by weight, Sigma Chemical Co., St. Louis, MO) were prepared in distilled water at $\sim 80^{\circ} \mathrm{C}$. Stock solutions of the analyte compounds were prepared, and serial dilutions were used to generate standards of various concentrations. Aliquots of the standard solutions were then added to the gelatin solutions. Concentrations were calculated on the basis 
of dry weight for both the gelatin and analyte compounds. For each compound studied, six samples were prepared with concentrations ranging from $1 \mathrm{ppm}$ to $10 \%$ by weight. Compounds used in this study were obtained commercially and used without further purification. The spin-cast doped gelatin films were prepared by depositing one drop of the gelatin/compound mixture (while still warm and not too viscous) onto a $1-\mathrm{cm} \times 1-\mathrm{cm}$ silicon wafer slice that was spinning at $12,000-15,000 \mathrm{rpm}$ on a May Spinning Disk Aerosol Generator (BGI Inc.r Waltham, MA).* Spincasting of the gelatin solutions results in a flat, homogeneous film that completely covers the silicon substrate. Film thicknesses, as measured with a surface profilometer, were found to be $30-50 \mathrm{~nm}$. This was sufficiently thin to eliminate the need for charge compensation during SIMS analysis. Thicker films can be prepared by reducing the spin rate or increasing the viscosity of the mixture. Films prepared in this manner appear very robust and could be analyzed several months later with no apparent degradation.

The films were analyzed on two different types of SIMS instrumentation. For many of the studies, a commercially available Cameca IMS-3F or 4F ion microscope was used. The gelatin films were analyzed using an $8.0-\mathrm{keV} \mathrm{Ar}^{+}$beam rastered over an area of 500 $\mu \mathrm{m} \times 500 \mu \mathrm{m}$, with detection of positive secondary ions. The instrument can be operated under static (primary ion dose less than or equal to $1 \times 10^{13}$ atoms $/ \mathrm{cm}^{2}$ ) or dynamic (primary ion dose greater than $1 \times 10^{13}$ atoms $/ \mathrm{cm}^{2}$ ) analysis conditions. Unless otherwise stated, analyses were performed under $d y$ namic analysis conditions, with a total primary dose exceeding $1 \times 10^{15}$ ions $/ \mathrm{cm}^{2}$. At this dose, the parent compound was completely consumed or fragmented. Working curves were constructed by taking the integrated areas under the signal decay curves for the parent molecule (normalized to a constant chemical background signal level) and plotting them as a function of analyte concentration in units of grams of compound per gram of gelatin matrix. For secondary ion imaging studies, images were obtained with a scientific-grade slow-scan charge-coupled device (CCD) camera (Star 1; Photometrics Inc., Tucson, AZ). The sample chamber pressure of the Cameca IMS $4 \mathrm{~F}$ was $\sim 3-4 \times 10^{-10}$ torr, and introduction of the gelatin film samples did not result in any significant increase in this base pressure.

A time-of-flight (TOF) SIMS mass spectrometer was also used to examine the gelatin films. This instrument was constructed at the Naval Research Laboratory (NRL) and is discussed in detail elsewhere [8]. The instrument utilizes a pulsed-ion gun containing a

\footnotetext{
"Certain commercial equipment, instruments, or materials are identified in this report to specify adequately the experimental procedure. Such identification does not imply recommendation or endorsement by the National Institute of Stardards and Technology, nor does it imply that the materials or equipment identified are necessarily the best available for the purpose.
}

thermionic emitter source that produces 2-5-ns (full width at half-maximum) pulses of $13.0-\mathrm{keV} \mathrm{Cs}^{+}$ions. These primary ions bombard the sample, which is held at the secondary accelerating potential of $\pm 5 \mathrm{keV}$, resulting in a primary ion impact energy of 18.0 and $8.0 \mathrm{keV}$ for negative and positive secondary ions, respectively. The mass analyzer consists of a $60-\mathrm{cm}$ flight path into a reflection electrostatic mirror where the secondary ions are tocused and retlected along the flight path to a dual-channel plate detector. The primary ion beam had an average continuous current of $12 \mathrm{nA}$ and bombarded a sample area of approximately $3 \mathrm{~mm}^{2}$. This resulted in a total primary dose during an analysis of $3 \times 10^{6}$ cycles ( $3 \mathrm{~ns} /$ cycle) of approximately $2 \times 10^{10}$ ions $/ \mathrm{cm}^{2}$, well within the static SIMS limit. Working curves were established by plotting the integrated intensity of the parent ion peak relative to the integrated intensity of $\mathrm{m} / z 73$ (a constituent of the gelatin matrix) as a function of concentration.

\section{Results and Discussion}

\section{Analysis of Doped Gelatin Films}

As shown in Table $1 \mathrm{a}$ number of different types of compounds were prepared in the gelatin matrix. These films were analyzed in both SIMS instruments, with similar working curves and detection limits obtained from each. Figure 1 shows the response curve for tetrabutylammonium bromide as a function of concentration as acquired on the TOF SIMS instrument. Similarly, Figure 2 shows the curve for benzalkonium chloride (a germicide used in many personal care products), also obtained on the TOF SIMS instrument. The working curves for these compounds exhibit nonlinear behavior as a function of increasing concentration (for comparison, a theoretical linear signal response with increasing concentration is shown by a dotted line). These effects are discussed later. For both of these compounds, detection limits were $\sim 1 \mathrm{ppm}$ (the detection limit was defined as the concentration at which the integrated analyte signal is at least two times the discrete chemical background). These detection limits are well within the range of what would be considered physiologically relevant. It should be noted that the detection limit data were by no means optimized, and the numbers reported should be considered upper

Table 1. Detection limits for compounds in gelatin films

\begin{tabular}{ll}
\hline Compound & $\begin{array}{l}\text { Detection limit range } \\
\text { (g compound/g gelatin) }\end{array}$ \\
\hline \hline Acetylcholine & $0.001-0.01$ \\
Epinephrine & $0.01-0.1$ \\
Cocaine & $10^{-4}-10^{-3}$ \\
Tetrabutylammonium bromide & $1 \times 10^{-6}$ \\
Tetrapentylammonium bromide & $0.2-2 \times 10^{-6}$ \\
Benzalkonium chloride & $1 \times 10^{-6}$ \\
Secobarbital & $10^{-4}-10^{-3}$ \\
Methylene blue & $10^{-4}-10^{-3}$ \\
\hline
\end{tabular}




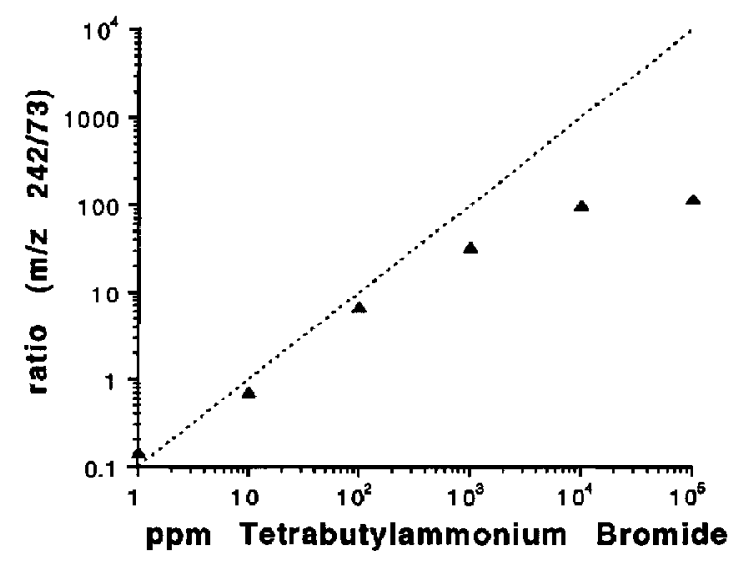

Figure 1. Response curve for tetrabutylammonium bromide as a function of concentration in gelatin, as acquired on the NRL TOF SIMS instrument. To highlight deviations from linear behavior, a theoretical linear signal response with increasing concentration is shown by the dotted line.

limits. It has recently been shown that by appropriate selection of primary ion energy, species, and impact angles, improvements can be made in the number of intact molecules detected in an organic SIMS experiment [1]. Other compounds, such as cocairı- hydrochloride, shown in Figure 3 (ion microscope data), exhibited lincar working curves but had much poorer detection limits. This probably reflects the lower ionization yield for protonated molecular ions. A summary of detection limit ranges for the compounds examined is presented in Table 1 (in this case we define the detection limit range as the range in concentration in which the analyte signals fall to two times the discrete chemical background).

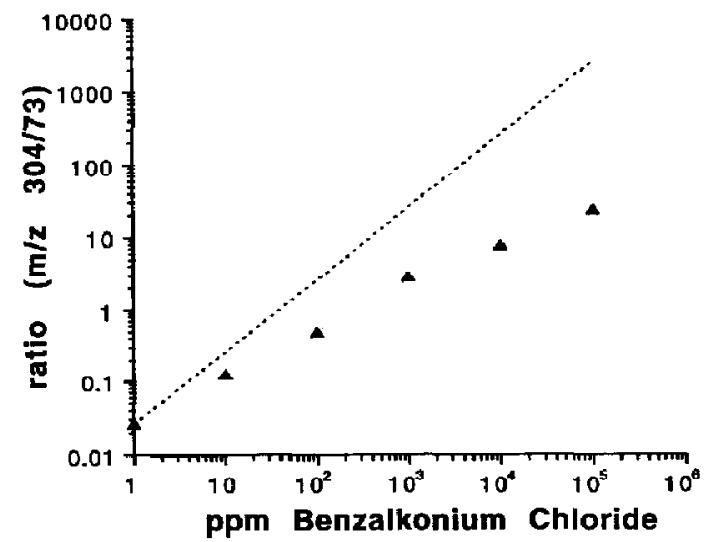

Figure 2. Response curve for benzalkonium chloride (a germicide used in many personal care products) as a function of concentration in gelatin, as acquired on the NRL TOF SIMS instrument. To highlight deviations from linear behavior, a theoretical linear signal response with increasing concentration is shown by the dotted line.

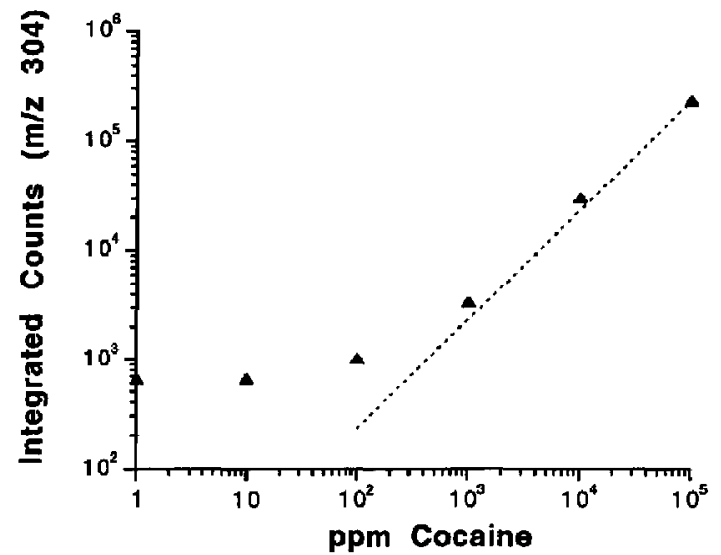

Figure 3. Response curve for the protonated molecular ion of cocaine as a function of concentration in gelatin, as acquired on the ion microscope. No saturation in signal is observed at higher analyte concentrations.

\section{Film Homogeneity}

Evaluation of the homogeneity of the gelatin films was not possible with the NRL TOF SIMS because the position of the sample is fixed, preventing multiple analyses at different positions. To check the homogeneity a brief study was conducted with another $\mathrm{TOF}$ SiMS instrument (Kratos 4OIt S; Kratos, Ramsey, NJ) using $25-\mathrm{keV}$ gallium bombardment of a $700-\mu \mathrm{m} \times$ $700-\mu \mathrm{m}$ square area at various positions on the sample. In this case the spot-to-spot reproducibility for the normalized signal intensity was better than $10 \%$. Using the ion microscope in the static analysis mode (primary dose less than $1 \times 10^{13}$ ions $/ \mathrm{cm}^{2}$ ), with an analysis area of $500 \mu \mathrm{m} \times 500 \mu \mathrm{m}$, also gave a spotto-spot reproducibility of $\sim 10 \%$ (in this analysis only integrated signal intensities were used without normalization to a reference mass). Under dynamic analysis conditions, the reproducibility of the integrated signal (normalized to the carbon matrix signal) in the ion microscope was worse, with an average variation for an individual sample of $\sim 30 \%$. The poor reproducibility under dynamic conditions may result from the rapid decay in parent molecular ion signal (complete consumption of the molecule occurs in $1-5 \mathrm{~min}$ ). In the ion microscope instrument, with higher current primary beams, there is a significant flux of neutrals and scattered primary ions that strike a region much larger than the area being analyzed. This process can lead to predamaging of analyte molecules in a supposedly virgin area of the sample before that area is analyzed. Multiple profiles on the same sample often show a systematic loss of signal in the initial, highest intensity portions of the signal decay curve. This can lead to a significant error in the integrated signal calculation. One advantage of using dynamic analysis conditions for the analysis of these gelatin films is that a modest improvement in signal (slightly less than a 
factor of 2) can be achieved by consuming all of the sample. This may prove beneficial for maximizing detection limits but has a commensurate loss in reproducibility.

\section{Chemical Background}

It is interesting to note that some of the lower mass compounds, notably the neurotransmitter acetylcholine, have very poor detection limits. This is thought to result in part from the presence of a discrete background arising from polyatomic cluster ions. This background can severely degrade detection limits for lower mass analyte compounds. The influence of this background can be seen in the ion microscope mass spectrum shown in Figure 4 for a $0.1-\mathrm{g} / \mathrm{g}$ (10 wt\%) acetylcholine/gelatin mixture. Even for a compound such as acetylcholine, which is considered to have a high ion yield, the detection limit range for the parent cation $(m / z 146)$ was between $0.001 \mathrm{~g} / \mathrm{g}(0.1 \mathrm{wt} \%)$ and $0.01 \mathrm{~g} / \mathrm{g}(1 \mathrm{wt} \%)$. Similar poor detection limits were obtained for other lower mass compounds, such as epinephrine and tetraethylammonium bromide. Figure 5 shows the mass spectrum of a gelatin blank acquired on the TOF SIMS instrument, illustrating that the background extends to better than $1000 \mathrm{u}$. Over the mass range of the compounds examined (100-300 u), we find that compounds with higher molecular weights give better detection limits. This is partially due to the reduced chemical background but also, in the case of the quaternary salt molecules, to the ion yield enhancement, which occurs as the size of the cation increases (with increased cation size, the attractive coulombic force between the cation and anion is decreased, making it easier to separate the two counterions and increasing the observed secondary ion signal) [9]. Although we have not explored them in this study, there are several approaches that could be used to reduce the influence of the discrete chemical back-

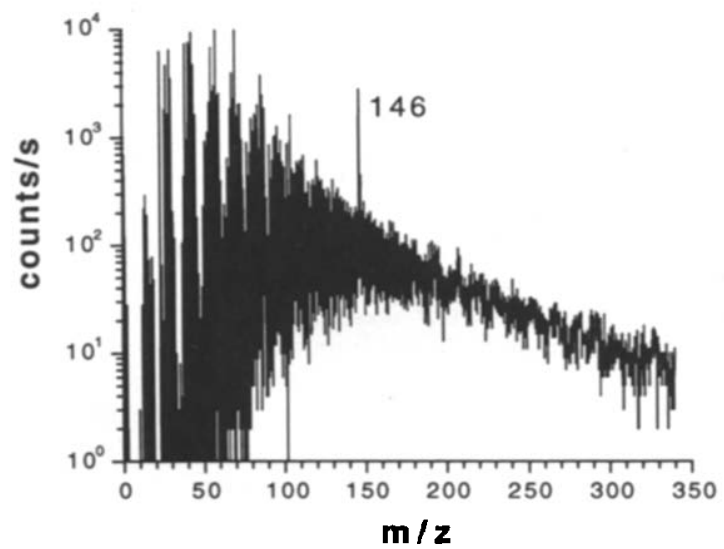

Figure 4. Mass spectrum from $0.1 \mathrm{~g} / \mathrm{g}$ acetylcholine in gelatin standard showing the influence of background on detection limit in the ion microscope.

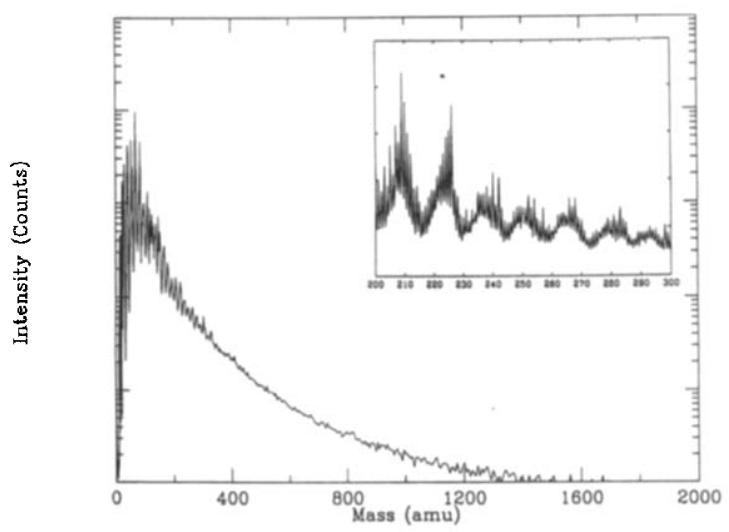

Figure 5. Mass spectrum for a blank gelatin film obtained on the TOF SIMS instrument demonstrating the chemical background.

ground. These include the use of high mass resolution [6], selective energy filtering [10], and tandem mass spectrometry SIMS imaging [11].

\section{Matrix Effects}

We often observe nonlinear working curves fur many of the quaternary ammonium salt compounds that we have examined. This effect is characterized by a saturation in secondary ion signal at increasing analyte concentrations (see Figure 1). This effect has not been observed for compounds that form protonated molecular ions. The variation from linearity also seems to depend on the quaternary compound being analyzed, with some compounds, such as tetrapentylammonium bromide (Figure 6), showing nonlinear behavior only at the highest concentrations. Although the origin of this effect is not clear, it is possible that the saturation in signal may be due to a higher probability for reneutralization with increasing counterion concentration

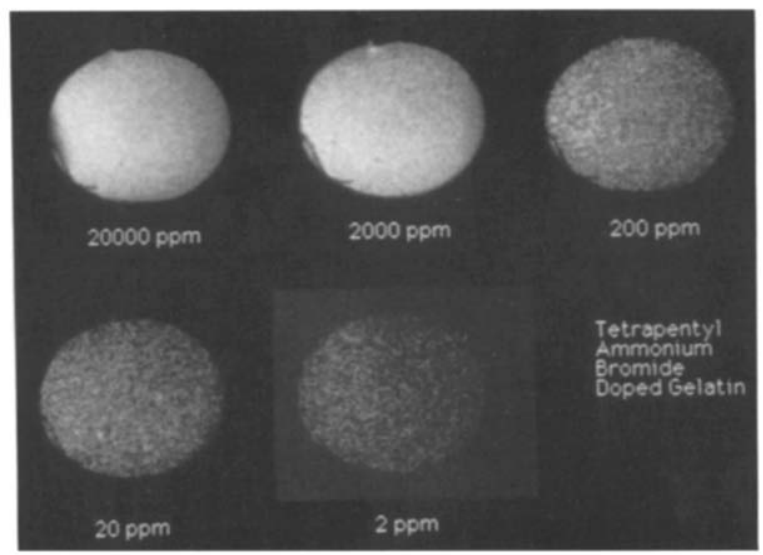

Figure 6. Series of secondary ion images from tetrapentylammonium bromide doped in gelatin as a function of concentration. 
[12]. In any case the observation of molecular matrix effects suggests that care will have to be taken in the interpretation of quantitative data from real samples in which localized analyte molecular concentrations (or localized counterion concentrations) may result in smaller secondary ion signals than from adjacent regions where the true concentration is actually lower. It might be expected that similar effects may occur for cationized molecular species, such as $[\mathbf{M}+\mathbf{K}]^{+}$and $\left[\mathrm{M}+\mathrm{Na}^{+}\right.$. In this case it will be important to determine whether the signal reflects the composition of the analyte molecule or local variations in the concentration of the adduct species. Clearly, for quantitative interpretation these questions are important.

\section{Quantitative Molecular Imaging Standards}

One of the most intriguing aspects of SIMS is its potential ability to generate images of the spatial distribution of organic molecules in biological systems [1-3]. One of the initial goals of the present study was to develop standards that could potentially be used to quantify (on an individual pixel basis) an image from a molecular species in an unknown sample. In addition to bulk analysis, the homogeneity of the doped spincast films could make them useful as standards for quantitative molecular imaging SIMS studies. A tetrapentylammonium bromide gelatin film was analyzed using a slow-scan CCD camera imaging system as the primary detector (shown in Figure 6). Signal intensities were determined by integrating the total secondary ion counts in each image. The resulting working curve is displayed in Figure 7 . Because of its high linearity and high dynamic range, the $C C D$ camera is ideally suited for these studies, and working curves were obtained over a concentration range $2 \times 10^{-5}-0.02 \mathrm{~g} / \mathrm{g}$.

\section{Conclusions}

Spin-cast gelatin films are useful as model matrices for quantitative organic SIMS studies. The spin-casting procedure is simple to implement, and the films are superior to previous methods of preparation and eliminate many of the thickness and charging problems that were typically encountered. Analysis of these films by SIMS demonstrated that for many compounds (particularly salts), detection limits of $1 \mathrm{ppm}$ are possible. Also, their homogeneity makes them suitable as stan-

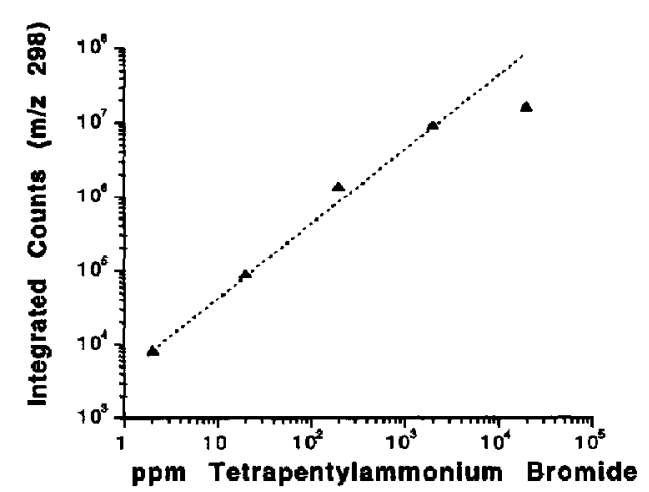

Figure 7. Working curve generated from the integrated counts in each image of Figure 6 . To highlight deviations from linear behavior, a theoretical linear signal response with increasing concentration is shown by the dotted line.

dards for quantitative molecular imaging studies. The gelatin films may prove useful for fundamental studies of molecular desorption from biological matrices, allowing us to study matrix effects and ionization phenomena.

\section{References}

1. Gillen, G.; Simons, D. S.; Williams, P. Anal. Chem. 1990, 62 , 2121.

2. Lindley, P. M.; Chakel, I. A.; Odom, R. In Secondary Ion Mass Spectrometry: SIMS 8; Benninghoven, A.; Janssen, K. T. F.; Tumpner, J.; Werner, H. W.; Eds.; Wiley: Clucilester, 1992, $\mathrm{p}$ 218.

3. Todd, P. J;; Short, T. Proceedings of the 40th ASMS Conference on Mass Spectrometry and Allied Topics; Washington, DC. May-June 1992; p 1961.

4. Burns-Bellhorn, M.; File, D. M. Anal. Biochem. 1979, 92, 213.

5. Burns, M. S. J. Microsc. 1981, 127, 237.

6. Zhu, D.; Harris, W. C.; Morrison, G. H. Anal. Chem. 1982, 54, 1963.

7. Ramseyer, G. O.; Morrison, G. H. Anal. Chem. 1983, 55, 1963.

8. Hues, S. M.; Colton, R. J.; Mowery, R. L.; MeGrath, K. J.; Wyatt, J. R. Appl. Surf. Sci. 1988-1989, 35, 507.

9. Kidwell, D.; Ross, M. M.; Colton, R. J. Int. J. Mass Spectrom. lon Processes 1987, 78, 315.

10. Gillen, G. Int. J. Mass Spectrom. Ion Processes 1991, 105, 215.

11. Todd, P. J.; Short, R. T.; Grimm, C. S; Holland, W. M.; Markey, S. P. Anal. Chem. 1992, 64, 1871.

12. Williams, P.; Gillen, G. In Ion Formation From Organic Solids: IFOS IV; Benninghoven, A., Ed.; Wiley: Chichester, 1989; p 15. 